\title{
Timor-Oriental : le combat pour la paix et pour la réconciliation
}

Rodd McGibbon et Roland Bleiker

\section{(2) OpenEdition \\ 12 Journals}

Édition électronique

URL : http://journals.openedition.org/conflits/401

DOI : $10.4000 /$ conflits.401

ISSN : $1777-5345$

Éditeur :

CCLS - Centre d'études sur les conflits lilberté et sécurité, L'Harmattan

Édition imprimée

Date de publication : 4 mars 2001

ISBN : 2-7475-0725-4

ISSN : 1157-996X

\section{Référence électronique}

Rodd McGibbon et Roland Bleiker, «Timor-Oriental : le combat pour la paix et pour la réconciliation », Cultures \& Conflits [En ligne], 41 I printemps 2001, mis en ligne le 14 septembre 2006, consulté le 30 mars 2021. URL : http://journals.openedition.org/conflits/401 ; DOI : https://doi.org/10.4000/conflits. 401

Ce document a été généré automatiquement le 30 mars 2021.

Creative Commons License 


\title{
Timor-Oriental : le combat pour la paix et pour la réconciliation
}

\author{
Rodd McGibbon et Roland Bleiker
}

1 Timor-Oriental : le combat pour la paix et pour la réconciliation Roland BLEIKER et Rodd MCGIBBON ${ }^{1}$ Quelle est la part du passé dont il faut garder le souvenir et faire le

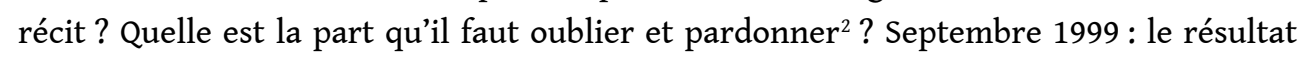
$\mathrm{du}$ référendum organisé au Timor-Oriental est très largement favorable à l'indépendance. Grâce à CNN, gouvernements et simples citoyens du monde entier assistent alors, scandalisés, aux exactions perpétrées par les milices antiindépendantistes. Très rapidement, le sort des Est-Timorais suscite sur toute la planète un élan de solidarité qui se traduit par l'envoi d'une force armée, baptisée Interfet, mandatée par l'ONU et placée sous commandement australien, avec la mission de rétablir l'ordre dans le territoire. Toutefois, en dépit de cette intervention réussie, les milices laissent derrière elles un territoire dévasté et une population en état de choc. Selon l'ONU, en effet, $70 \%$ de l'infrastructure du territoire a été détruite ${ }^{3}$. Officiellement, ces événements ont fait 600 morts, mais l'ONU elle-même reconnaît qu'il est possible que le nombre de morts ait été beaucoup plus élevét. De très nombreux habitants ont pris la fuite ou ont été expulsés par la force. La ville principale du Timor-Oriental, Dili, est en ruines, tout comme d'autres villes du territoire. Dans la ville d'Aileu, par exemple, "seuls l'église et le commissariat étaient encore debout; les 17000 habitants avaient fui $»^{5}$. Confrontés à ces destructions, les Est-Timorais s'attaquent à la tâche colossale de la re-con-struc-tion. L'Administration provisoire de l'ONU au Timor-Oriental (UNTAET) fut mise en place pour superviser le processus de reconstruction et pour préparer la transition vers l'in-dé-pen-dance. Mais maintenant que le combat pour la paix au Timor-Oriental est devenu quotidien plutôt qu'héroïque, les médias globaux ont délaissé Dili pour d'autres points chauds: des inondations catastrophiques au Mozambique, un coup d'Etat aux îles Fidji, une élection truquée au Pérou ou tout autre évé-ne-ment dramatique susceptible de retenir - brièvement l'attention volage des téléspectateurs mon-diaux. Au Timor-Oriental pourtant, le combat pour une paix durable ne fait que commencer. Au-delà même de la reconstruction physique, il s'agit de la recomposition, quotidienne et patiente, d'une 
société en état de choc. L'objet de cet article est d'explorer et d'analyser ces défis. Bien sûr, il est toujours difficile d'étudier dans leur complexité une situation d'urgence et ses conséquences $^{6}$. Aussi convient-il, avant même d'engager cet effort sur le fond, d'en reconnaître les limites. La pacification au Timor-Oriental prendra du temps et les obstacles auxquels elle devra faire face seront nombreux. Elle exigera, pour citer John Paul Lederach, "que l'on s'engage à long terme dans la mise en place d'une infrastructure, à tous les niveaux, qui mobilise toutes les ressources porteuses de réconciliation au sein de la société et qui maximise les soutiens extérieurs ${ }^{7}$. La pacification au Timor-Oriental ne peut se dérouler - et s'observer - qu'à long terme et à trois niveaux liés : ceux des communautés locales, de la nation est-timoraise et, enfin, de la relation de celle-ci avec l'extérieur. De ce dernier point de vue, la relation à l'Indonésie a une importance particulière, tout comme le rôle de la communauté internationale dans le processus de reconstruction. Dans la première partie de cet article, nous évoquerons les effets sur la société est-timoraise de la brutale occupation indonésienne ainsi que des exactions paramilitaires consécutives au référendum sur l'indépendance. Dans une deuxième partie, nous présenterons d'abord brièvement les principaux acteurs locaux, nationaux et internationaux de la pacification actuelle et future. Puis nous concentrerons notre attention sur les principaux problèmes actuels, avec un accent particulier sur la question de la participation locale au processus de restructuration. Enfin, dans une troisième et dernière partie, nous désignerons quelques-uns des principaux problèmes auxquels la construction d'une paix au TimorOriental devra faire face à long terme. Trois enjeux paraissent déterminants: le dialogue national, la mise en place d'institutions démocratiques et les relations avec l'Indonésie. Pour finir, une mise en garde. L'issue de toute initiative pour aider la population est-timoraise à surmonter son état de choc dépendra de très nombreux facteurs : de la portée pratique de certains principes démocratiques et humanitaires de base, certes, mais aussi, et surtout, des comportements qu'adopteront les principaux acteurs du conflit est-timorais. C'est pourquoi nous nous abstiendrons de recourir à un modèle universaliste de pacification. Cela reviendrait, en effet, à imposer de l'extérieur un ensemble de postulats qui ne permettraient vraisemblablement pas de prendre en compte la complexité de la situation à laquelle ce pays doit faire face. Au contraire, nous proposons d'étudier et d'évaluer la pacification à la lumière de dilemmes politiques et de relations sociales spécifiques. Cette démarche ne signifie pas pour autant que nous souhaitions proposer des solutions politiques concrètes aux problèmes quotidiens, tâche qui revient aux diplomates et aux responsables politiques. En fait, notre objectif principal est de nous confronter aux défis éthiques à venir, tout en appuyant cette confrontation sur une étude empirique approfondie des enjeux pratiques. Un territoire dévasté : l'héritage de l'occupation et du pillage Le TimorOriental subit la domination de l'étranger depuis des siècles. Au cours du XVIIe siècle, les rivalités coloniales furent à l'origine de la partition de l'île de Timor en deux moitiés : Est, sous contrôle portugais, et Ouest, sous contrôle néerlandais. La période de colonisation qui s'ensuivit aviva les vieilles rivalités tribales ou politiques qui divisaient déjà la société timoraise. Quand le Portugal décida, en 1975, de décoloniser le TimorOriental en retirant précipitamment ses troupes et son administration, ces rivalités éclatèrent au grand jour et déclenchèrent une brève guerre civile ${ }^{8}$. "Diviser pour régner ». L'occupation coloniale du Timor-Oriental par l'Indonésie Le Portugal avait mal préparé la décolonisation du Timor-Oriental et, en Indonésie, ce manque de préparation suscita immédiatement des inquiétudes quant à la sécurité du pays, 
notamment parmi les plus hauts responsables de l'armée. Aussi ces derniers chargèrent-ils leurs agents de déstabiliser le territoire. D'une manière générale, les chercheurs s'accordent à dire que cette déstabilisation du Timor-Oriental fut organisée pour offrir un prétexte à une future invasion du territoire, voire à son éventuelle annexion. Pourtant, que ce soit sur le plan religieux, sur le plan linguistique ou sur le plan historique, le Timor-Oriental a très peu en commun avec l'Indonésie bien que celle-ci soit déjà très hétérogène. Dès que l'Indonésie débuta l'occupation du TimorOriental, il apparut clairement qu'elle envisageait de contrôler le territoire par la force. Les troupes indonésiennes qui prirent part aux premières attaques contre la population est-timoraise n'avaient reçu qu'un entraînement rudimentaire et la férocité dont elles firent alors preuve donna le ton des années d'occupation qui allaient suivre. Du fait de cette stratégie brutale, seuls les plus chauds partisans est-timorais de l'intégration à l'Indonésie continuèrent à soutenir l'occupation'. L'Indonésie essaya d'asseoir sa domination sur le vieux principe colonial « diviser pour régner ». Les divisions au sein de la société est-timoraise furent donc exploitées dans le but de mettre un frein à l'émergence de mouvements de résistance. L'héritage funeste de l'occupation coloniale, dont le poids se fait encore sentir aujourd'hui, sera analysé ci-après. À ce stade, il nous semble plus important de souligner le fait que l'Indonésie ne pouvait pas se contenter de «diviser pour régner». Au Timor-Oriental, en effet, l'Indonésie avait également besoin d'un puissant appareil policier, qu'elle ne tarda pas à mettre en place ${ }^{10}$. Malgré tout, des mouvements de résistance localisée apparurent très vite. Comme la plupart des mouvements indépendantistes, ces mouvements s'enracinaient dans «le désir d'avoir "sa" terre $»^{11}$, qui associe généralement la révolte armée, qui vise le contrôle d'un territoire donné, à une lutte plus symbolique autour de l'identité et de la souveraineté. Le mot «territoire " rappelle d'ailleurs par son étymologie même qu'il porte en germe le conflit: sa racine est en effet le verbe latin terrere qui signifie "effrayer", "épouvanter", "faire fuir " ${ }^{12}$. Au Timor-Oriental, la résistance à l'occupation indonésienne prit tout d'abord la forme d'une guérilla essentiellement rurale. Avec l'extension de la lutte à l'agitation urbaine, qui concerna principalement des militants étudiants, l'activité des services secrets indonésiens se développa ${ }^{13}$. L'armée indonésienne mit ainsi en place un vaste réseau d'informateurs et d'espions dans le but de mettre un frein au développement des mouvements de résistance dans les villes. Cette montée en puissance du contre-espionnage et de la répression indonésiens fut dévastatrice : selon certaines sources, elle coûta la vie à environ 200.000 personnes - soit le quart de la population - massacrées ou emportées par la faim et par la maladie ${ }^{14}$. Pour contrôler le Timor-Oriental, l'Indonésie associa à la répression la promotion du « développement » du territoire, conçue pour se concilier la population. De fait, des sommes importantes furent mobilisées, au profit notamment du système éducatif ${ }^{15}$. Rapporté au nombre d'habitants, le budget que le gouvernement indonésien allouait au Timor-Oriental était d'ailleurs supérieur à celui de toutes les autres provinces. Il est vrai que le développement de l'infrastructure du territoire visait en partie à faciliter les opérations que l'armée indonésienne menait contre les mouvements de résistance; mais il faut reconnaître également que le gouvernement indonésien était convaincu que s'il contribuait au développement du Timor-Oriental, il affaiblirait du même coup les mouvements de résistance en question. La croissance économique du Timor-Oriental était peut-être le refrain que l'on chantait à Jakarta, mais la terreur que l'armée indonésienne faisait régner dans la population et les rivalités qui divisaient la société est-timoraise rendaient difficile la mise en œuvre d'un 
véritable développement. Des analyses détaillées ont montré, en effet, que l'essentiel des bénéfices de l'activité économique au Timor-Oriental allait à des allochtones, et notamment aux militaires ${ }^{16}$. Ces derniers ont détenu en particulier le monopole de la très lucrative filière du café. Puis, au cours des années quatre-vingt-dix, ce furent les enfants du Président Suharto qui commencèrent à contrôler ce type d'activités économiques, avec l'appui du gouverneur de la province du Timor-Oriental, Abilio Osorio Soares. Même aux échelons économiques les plus modestes, les Est-Timorais restaient désavantagés. Ainsi, c'étaient des immigrés originaires de lîle de Sulawesi qui contrôlaient l'essentiel de l'économie informelle du territoire, y compris les petits commerces. La plupart des personnes exerçant une profession libérale et la plupart des fonctionnaires, particulièrement les hauts fonctionnaires, étaient originaires de Java ou d'autres provinces en-dehors du Timor-Oriental. L'occupation indonésienne ôta tout pouvoir aux populations locales et, en le concentrant entre les mains de l'armée indonésienne et de la bureaucratie des administrations locales, fit obstacle au développement d'une société civile viable. Cette stratégie, parfaitement consciente, fut d'ailleurs appliquée dans la plupart des provinces indonésiennes. Les médias étaient très sévèrement censurés et les ONG devaient subir sans cesse les tracasseries et les manœuvres d'intimidation de l'armée indonésienne. Par ailleurs, la politique économique menée par l'Indonésie ne permettait pas l'émergence d'entrepreneurs locaux. Les rares Est-Timorais qui accédaient malgré tout à l'élite profitaient de leur accès privilégié aux ressources de l'Etat pour s'enrichir personnellement plutôt que pour promouvoir des activités entrepreneuriales indépendantes ${ }^{17}$. En son temps, le Portugal avait fait jouer un rôle essentiel aux chefs coutumiers dans le but d'asseoir indirectement son autorité coloniale, mais l'Indonésie n'adopta pas la même stratégie. $\mathrm{Au}$ contraire, les chefs coutumiers furent marginalisés par l'imposition d'un modèle uniforme d'administration publique (appelé Sistem Pemerintahan) ${ }^{18}$. Finalement, les membres des élites locales furent soit destitués, soit discrédités par les opposants à l'occupation indonésienne au titre de leur complicité avec l'autorité coloniale. Le rôle de la communauté internationale dans la légitimation de l'occupation indonésienne Il faut rappeler ici, fût-ce brièvement, que certains Etats ont été mêlés de très près à l'annexion du Timor-Oriental par l'Indonésie. En effet, alors même que l'Assemblée générale de l'ONU exigeait le retrait immédiat des troupes indonésiennes, les puissances occidentales apportaient un soutien tacite à l'opération : les considérations d'ordre idéologique liées à la guerre froide l'avaient emporté sur les violations des droits de la personne dont étaient victimes les Est-Timorais. Le régime militaire indonésien avait été mis en place dix ans plus tôt à la suite du massacre de plusieurs centaines de milliers de communistes et de présumés gauchistes. Bien que les EtatsUnis aient décrété un embargo sur les armes pendant quelques mois en 1966, ils s'abstinrent de condamner officiellement cette tuerie, que la CIA considère pourtant comme "l'un des plus grands massacres du XXe siècle ». Puis, très vite, les Etats-Unis commencèrent à nouer des liens militaires, économiques et politiques étroits avec le régime du président Suharto ${ }^{19}$. D'autres puissances occidentales leur emboîtèrent le pas. Le Royaume-Uni, les Pays-Bas et l'Allemagne de l'Ouest vendirent à l'Indonésie de grandes quantités de matériel de guerre et divers autres Etats occidentaux cherchèrent à leur tour à nouer avec elle des liens économiques. L'Australie fit preuve d'un empressement particulier. Elle fut en effet l'un des rares Etats à reconnaître officiellement l'annexion du Timor-Oriental par l'Indonésie. L'armée australienne assurait même l'entraînement de soldats indonésiens, qui étaient très souvent envoyés 
au Timor-Oriental; elle assura notamment l'entraînement des redoutables troupes d'élite baptisées Kopassus dont la triste réputation n'est plus à faire. C'est ainsi dans le contexte de la rivalité entre les deux superpuissances et de la guerre froide, qui furent à l'origine de diverses guerres par procuration en Asie du Sud-Est, que l'Indonésie devint un allié du camp américain, aussi bien sur le plan militaire que sur le plan économique. L'Indonésie - le "domino » qui ne tomba pas - devint le symbole de la possibilité d'enrayer l'avancée menaçante du communisme en Asie du Sud-Est. Le soutien occidental en fut la récompense, sans égard pour le bilan désastreux des droits de l'homme en Indonésie. Dans ce cas, comme dans de nombreuses autres guerres par procuration entre les deux superpuissances, on peut dire avec Michel Wieviorka que la guerre froide a fonctionné « comme facteur d'aveuglement sur les déterminants et les significations locaux de diverses expériences de violence $»^{20}$. Cependant, la situation changea du tout au tout avec l'écroulement de l'Union soviétique et la fin de la guerre froide. Les Etats occidentaux durent en effet faire face à une forte pression exercée par des opinions publiques hostiles au soutien qu'ils apportaient à des régimes autoritaires. L'enjeu idéologique ayant disparu, l'Indonésie fut donc fermement invitée à organiser un référendum sur le futur statut du Timor-Oriental. Or, à la surprise générale, le président intérimaire Bacharuddin Jusuf Habibie annonça soudainement que la population est-timoraise allait bel et bien être consultée. Tout le monde connaît la suite. Les exactions des milices anti-indépendantistes et le nouvel ordre international C'est dans ce contexte que, fin 1988, l'armée indonésienne prit l'initiative de la constitution de milices anti-indépendantistes au Timor-Oriental. En fait, l'armée indonésienne avait noué des liens avec des Est-Timorais favorables à l'intégration du Timor-Oriental à l'Indonésie depuis les années soixante-dix. L'armée indonésienne utilisait ceux-ci dans le cadre de ses activités de renseignement, mais elle avait également recours à eux pour former des groupes paramilitaires supplétifs des troupes régulières dans le combat contre les «Forces armées de libération nationale du TimorOriental » (Falintil). Il était difficile de faire la distinction entre l'armée indonésienne et ces groupes paramilitaires, particulièrement dans le cadre d'opérations secrètes menées par l'armée indonésienne contre la guérilla indépendantiste. Les Forces spéciales, généralement à la tête des opérations secrètes, intensifièrent leurs activités à partir du début des années quatre-vingt-dix. C'est à cette époque que le colonel Prabowo, gendre de Suharto qui devait devenir par la suite général de division, en prit le commandement. La tactique du colonel Prabowo consistait notamment à faire appel à des groupes paramilitaires - souvent appelés "ninjas » - telle la Garda Paksi ou "Jeune garde pour le maintien de l'intégration", afin de faire pression sur la population en la terrorisant ${ }^{21}$. Aussi, lorsque le gouvernement indonésien annonça qu'il allait organiser un référendum sur le futur statut du Timor-Oriental, les responsables de l'armée se tournèrent-ils vers leurs alliés traditionnels pour montrer au monde entier que la population est-timoraise souhaitait en réalité que le Timor-Oriental demeure une province de l'Indonésie. Et, en effet, les membres et les dirigeants des milices qui commencèrent à se mettre en place à partir de décembre 1998 étaient essentiellement des Est-Timorais qui avaient coopéré avec l'armée indonésienne au cours des décennies précédentes. Les deux milices les plus connues - Halilintar et Aitarak - étaient dirigées respectivement par Joao da Silva Tavares et Eurico Guteres. Da Silva Tavares s'était vu confier la direction du district de Bobonaro en récompense de son soutien à la cause anti-indépendantiste et Guteres était un ancien commandant de la Garda Paksi. Les milices disposaient de bastions dans l'ouest du Timor-Oriental et, 
au cours de l'année 1999, ils étaient en mesure de contrôler par la terreur des districts entiers. Avant le référendum, l'objectif des milices était de montrer qu'une part importante de la population est-timoraise souhaitait que le Timor-Oriental demeure une province de l'Indonésie. Pour atteindre cet objectif, ces groupes organisèrent de grandes manifestations dans leurs bastions et exercèrent des pressions sur la population. Cette stratégie remporta un certain succès faute d'une élite locale crédible. De plus, comme rien n'était mis en place à ce niveau pour circonscrire et résoudre les conflits, les milices pouvaient recourir à la violence comme bon leur semblait afin de s'opposer à ceux qui, toujours plus nombreux, réclamaient l'indépendance du TimorOriental. On pourrait s'étonner des relations réduites entretenues par ces groupes avec la petite partie de l'élite de la société est-timoraise qui vivait à Dili ou à Jakarta, pourtant favorable, elle aussi, à l'intégration du Timor-Oriental à l'Indonésie. Cela s'explique, comme nous l'avons vu précédemment, par le fait que les milices étaient l'émanation de la seule armée indonésienne et plus particulièrement des tristement célèbres Kopassus. C'est d'ailleurs cette raison plus que toute autre qui explique l'échec de la médiation «Dare Dialogue » («osez le dialogue ») organisée en 1998 sous l'égide de l'Église catholique. En effet, l'objectif de cette médiation était de réconcilier par le dialogue l'élite est-timoraise favorable à l'intégration du Timor-Oriental à l'Indonésie avec les partisans de l'indépendance. Elle ne prenait ainsi en compte ni la destitution des élites locales, ni les collusions qu'elle favorisa entre l'armée indonésienne et les petits chefs de guerre est-timorais. Le sentiment qu'une crise était imminente commença à se faire jour lorsqu'il devint évident que la médiation échouait à inclure les groupes les plus radicaux. Toutes les conditions étaient réunies pour qu'un conflit éclatât : c'est ce qui se produisit dès que le résultat du référendum du 31 août 1999 fut connu et qu'il fut clair que l'écrasante majorité des Est-Timorais ne souhaitait pas que le Timor-Oriental demeure dans le giron de l'Indonésie. Les milices mirent alors le territoire à feu et à sang, mais une force armée multinationale, mise sur pied en un temps record, parvint à mettre un terme à leurs exactions. En fait, la rapidité de la réaction internationale traduisit l'importante évolution des relations internationales depuis la fin de la guerre froide. En effet, les Etats occidentaux commencèrent à abandonner leur soutien à des régimes anti-communistes répressifs et à lui substituer une diplomatie des droits de l'homme favorable à la réforme démocratique et libérale. Cette nouvelle diplomatie paraissait certes plus «éclairée ", mais il est probable que le changement était plus motivé par de nouvelles contraintes d'ordre idéologique ou géopolitique que par une soudaine conscience humanitaire. Une chose est sûre cependant : les manifestations qui eurent lieu dans le monde entier pour demander une intervention armée au Timor-Oriental et les précédents en Somalie, en BosnieHerzégovine et au Kosovo, ont largement contribué à ce qu'une action internationale soit organisée avec promptitude. Une force armée multinationale placée sous commandement australien débarqua donc au Timor-Oriental et parvint à y rétablir l'ordre. Malheureusement, les ravages étaient déjà considérables : plusieurs centaines d'Est-Timorais avaient trouvé la mort et plusieurs centaines de milliers d'autres avaient dû abandonner leur foyer ${ }^{22}$. La société est-timoraise, qui avait déjà dû subir des décennies d'occupation armée, se trouvait désormais en état de choc profond. Le défi du Timor-Oriental aujourd'hui: la reconstruction Étant donnée l'ampleur des destructions qu'a connues le Timor-Oriental, la reconstruction va demander un effort considérable et soutenu. Bien sûr, la toute première tâche était humanitaire : rétablir l'ordre et mettre des abris et de la nourriture à la disposition des personnes déplacées. 
Mais, les troupes de l'Interfet ayant rétabli l'ordre, il fallut se préoccuper du processus de reconstruction à long terme. C'est alors que l'UNTAET (Administration provisoire de l'ONU au Timor-Oriental) entre en scène. Son rôle est fondamental parce que, outre sa mission de coordination de la reconstruction, elle dispose de moyens et d'une légitimité essentiels à une pacification effective du Timor-Oriental. Mais avant d'examiner plus en détails ce processus, il convient de présenter quelques-uns des principaux acteurs qui vont modeler les défis politiques à venir. Les principaux acteurs La principale organisation politique du Timor-Oriental est le Conselho Nacional da Resistencia Timorense (CNRT), coalition des anciens partis politiques est-timorais, dont le Fretilin. Ce dernier est l'un des plus influents partis politiques favorables à l'indépendance du Timor-Oriental et il fut l'un des principaux protagonistes de la brève guerre civile qui éclata en 1975. Le CNRT quant à lui a été créé en 1997 dans le but de rapprocher les partis politiques décidés à faire front contre l'Indonésie. Né dans ce cadre nationaliste, le CNRT en tire, compte tenu des événements ultérieurs, une crédibilité morale qui lui permet de revendiquer un leadership politique. Sa légitimité historique lui donne un rôle important dans l'unification nationale et la construction au Timor-Oriental d'une culture politique commune. De fait, sous la direction de José Xanana Gusmão, le CNRT bénéficie d'un large soutien populaire. Aussi est-il probable que cette organisation sera appelée à servir de base à l'infrastructure politique qui devra être mise en place au Timor-Oriental au cours de la transition vers l'indépendance. Comme nous l'avons vu, le Falintil fut l'aile militaire de la résistance à l'occupation indonésienne. Cette armée de guérilla ne comptait guère que trois cents soldats au milieu des années quatre-vingtdix, mais son effectif augmenta au cours des années 1998 et 1999 au fur et à mesure que les appels en faveur de l'indépendance du territoire se faisaient plus pressants. Notons au passage que le Falintil s'est montré remarquablement discipliné lorsque Gusmão, alors à son commandement, lui a ordonné de ne pas répondre aux provocations des milices anti-indépendantistes alors même que les exactions de ces derniers battaient leur plein. Sans cette retenue, en effet, le Timor-Oriental aurait pu être plongé dans une véritable guerre civile. Sans doute une fraction significative de la population fut-elle déplacée, mais le nombre de victimes aurait certainement été beaucoup plus élevé si la guérilla s'était attaqué aux milices, fortement armées, ou a fortiori à l'armée indonésienne. Une fois que le combat qu'ils menaient contre l'occupation indonésienne fut achevé, les combattants du Falintil quittèrent les camps qu'ils avaient installés dans les montagnes. Mais, jusqu'à présent, les responsables de l'UNTAET ont évité pour l'essentiel de se préoccuper de leur démobilisation pour ne pas créer de tension avec les dirigeants est-timorais. Par ailleurs, les responsables de l'Untaet sont conscients que le Falintil est la seule organisation à disposer de l'autorité nécessaire pour maintenir l'ordre dans le territoire tant qu'un système judiciaire viable n'aura pas été mis en place. Cependant, si les combattants du Falintil ne sont pas contrôlés, ils risquent d'être tentés de rendre eux-mêmes une justice sommaire et d'abuser, plus généralement, de leur pouvoir. De fait, on signale ici et là des cas d'intimidation des populations de la part d'anciens combattants du Falintil, appelés Oknum (renégats) ${ }^{23}$. La grande majorité des Est-Timorais pratiquant la religion catholique, l'Église est-timoraise est naturellement amenée à jouer un rôle de force unificatrice, qu'il s'agisse de la reconstruction de la nation ou de la mise en place d'un Etat démocratique. Il est même tout à fait possible que le rôle et la légitimité de l'Église se révèlent essentiels pour l'avenir du Timor-Oriental. Monseigneur Belo, lauréat du prix Nobel de la paix 1996, a fait preuve ces dernières années, comme Gusmão, de grandes qualités de leadership : il 
jouit aujourd'hui d'une forte autorité morale auprès de la communauté catholique esttimoraise. Il semble qu'il y ait eu des tensions, en 1998, entre Gusmão et Belo, qui paraissait prêt à s'engager de manière par trop politique. Depuis lors, cependant, Belo a plutôt cultivé l'image d'un médiateur au-dessus de la mêlée. En fait, pour que l'Église puisse jouer son rôle symbolique de force unificatrice - rôle essentiel à la pacification il est indispensable qu'elle garde une certaine indépendance à l'égard des questions politiques particulières. Les ONG est-timoraises joueront également un rôle déterminant aussi bien dans la transition vers l'indépendance du Timor-Oriental qu'une fois celle-ci réalisée. Le Yayasan Hak (Institut des Droits de l'Homme), le Fokurpers (Forum des Femmes pour la Communication) et la CDHTL (Commission des Droits de l'Homme du Timor-Oriental) sont trois des plus importantes d'entre elles. La plupart des ONG est-timoraises sont basées à Dili, mais elles opèrent dans la plupart des régions du territoire. Ces organisations mènent des projets de développement local, elles observent et elles dénoncent les violations des droits de la personne, elles aident les victimes de ces violations et elles proposent des programmes d'éducation civique. Malheureusement, le peu d'infrastructure que ces organisations avaient réussi à mettre en place a été détruit au cours des pillages de l'automne 1999. Aussi les militants et les organismes donateurs devront-ils fournir un effort considérable pour reconstruire puis pour améliorer les capacités d'action des ONG est-timoraises. Comme l'Église, les ONG sont une composante essentielle de la société civile - composante essentielle, à son tour, d'un véritable Etat démocratique. Bien souvent, en effet, les sociétés postcoloniales libérées par une guerre d'indépendance ont subi la dictature d'un parti unique ou d'autres formes de régime autoritaire. L'espoir de voir naître une société plus équitable s'envolait ainsi avec la substitution de formes de domination locales aux structures du pouvoir colonial. Dans le cas du Timor-Oriental, une Église indépendante au sein d'une société civile active pourrait donc constituer une force d'équilibre nécessaire au maintien d'une culture politique démocratique et pluraliste. Si les acteurs nationaux ont un rôle essentiel à jouer dans le processus de reconstruction, les forces internationales ne seront pas moins importantes. En plus de l'UNTAET, dont nous avons déjà parlé, les acteurs internationaux les plus influents sont le Fonds monétaire international, la Banque mondiale, des ONG internationales, des institutions spécialisées de l'ONU et certains gouvernements étrangers. Nous examinerons plus en détail le rôle particulier de certains de ces acteurs dans la suite de cette deuxième partie, mais il nous faut déjà signaler qu'ils ont des intérêts très divergents qui s'inscrivent dans un ensemble étonnamment disparate de préoccupations nationales et internationales: les politiques étrangères, souvent peu compatibles, d'Etats tels que l'Indonésie, l'Australie, le Portugal et les Etats-Unis; mais aussi les priorités organisationnelles des agences multilatérales et les objectifs commerciaux des détenteurs de capitaux étrangers. En fait, comme la société est-timoraise est complètement désorganisée, des intérêts étrangers financièrement puissants pourraient aisément prendre le pas sur les intérêts des populations, et, si elles sont mal contrôlées, contribuer de surcroît à aviver les rivalités en son sein. Les tensions qui se font jour et la nécessité d'associer les populations locales est-timoraises au processus de reconstruction À la fin de l'année 1999, l'ONU et la Banque mondiale ont organisé ensemble une "Mission commune d'évaluation" qui a tracé les grandes lignes d'un programme de reconstruction du Timor-Oriental. Dans le cadre de ce programme, une vingtaine d'organismes donateurs internationaux ont promis de verser un minimum de 522 millions de dollars au cours des trois premières années ${ }^{24}$. Le rapport prévoit en 
effet un effort de reconstruction à tous les niveaux qui mobilise des acteurs divers, aussi bien est-timorais qu'internationaux. Dans la troisième partie de cet article, nous étudierons les défis que le processus de reconstruction du Timor-Oriental devra relever pour parvenir à mettre en place une paix qui dure. Auparavant, il convient d'identifier les acteurs les plus importants de ce processus ainsi que les intérêts qu'ils défendent. Le rapport établi par la Mission commune d'évaluation prévoit que le processus de reconstruction du Timor-Oriental repose sur le principe d'une association aux décisions de l'ensemble des acteurs. Il faut reconnaître que cette intention est tout à fait louable ${ }^{25}$. De manière implicite en effet, ce rapport reprend à son compte une idée préconisée par de nombreux spécialistes de la pacification : que c'est au niveau des relations interpersonnelles et des interactions les plus quotidiennes que peut surgir un potentiel positif de transformation. Comme le dit Lederach, « lorsque l'on a affaire à un conflit prolongé et violent, toute transformation exige un travail de soutien aux personnes choquées et endeuillées; mais aussi un traitement des sentiments profonds de peur, de colère et de rancœur qui résultent du deuil, personnel ou familial $»^{26}$. Le rapport de la Mission commune d'évaluation a beau préconiser la participation, plus le temps passe, plus on est en droit de se demander dans quelle mesure ce sera suivi d'effet. Pour le moment, l'approche proposée est dépourvue du programme stratégique détaillé indispensable à la promotion d'un développement à la base [grass-roots]. Ainsi, le rapport désigne huit domaines prioritaires, parmi lesquels la « décentralisation du pouvoir » [community empowerment], ainsi que la santé, l'éducation ou l'agriculture. Il est recommandé à cet égard de mettre en place des " conseils de village ", afin de poser les fondements d'une gouvernance décentralisée à laquelle les populations puissent demander des comptes. Malheureusement, ni la façon dont ces conseils de village pourraient (ou devraient) aborder les problèmes relatifs aux sept autres domaines, ni les rapports de ces conseils avec les dynamiques plus larges de la société civile, ne semblent avoir fait l'objet d'une véritable réflexion. En cloisonnant ainsi la participation, distinguée des domaines concrets d'action publique, l'ONU et la Banque mondiale risquent de transformer la reconstruction en un processus hiérarchisé auquel les populations locales seront peu associées. De fait, au vu du bilan des organisations internationales, on peut craindre qu'elles se contentent d'emprunter le langage des partisans d'un développement humaniste et démocratique [people-centred], sans en prendre au sérieux les implications. Différents commentateurs critiques ont souligné que, en dépit de leur nouveau langage, ces institutions continuent de s'appuyer sur une approche formelle du développement dans laquelle la participation n'intervient que de façon circonstancielle et accessoire ${ }^{27}$. Si les populations est-timoraises ne sont pas suffisamment associées à la reconstruction, il est possible que les divisions sociales s'en trouvent avivées, ou que l'émergence de nouvelles tensions nourrisse de nouveaux conflits. Bien sûr, le passage à la démocratie ne suffit jamais, en lui-même, à faire disparaître les tensions existantes. En l'absence d'une véritable participation, cependant, la reconstruction ne sera ni contrôlable ni transparente. Or, si ces deux conditions essentielles ne sont pas réunies, la reconstruction risque d'être confisquée par des intérêts particuliers. Cela pourrait susciter un mécontentement populaire, voire des réactions violentes de la part de groupes qui se sentiraient exclus. L'empoignade autour des fonds alloués au développement du territoire, qui a déjà débuté, désigne clairement ces risques. Ainsi, des troubles ont éclaté après que l'UNTAET aie fait part de son intention d'embaucher des Est-Timorais, en raison de disputes violentes sur l'attribution de ces emplois ${ }^{28}$. Par ailleurs, les tensions pourraient 
encore s'accroître si la conduite de la reconstruction était accaparée par des consultants internationaux à forte rémunération, à l'exclusion des responsables esttimorais. Il faut reconnaître cependant que le dirigeant de l'UNTAET, Sergio Viera De Mello, a su en partie dissiper cette crainte en consultant régulièrement les dirigeants est-timorais. Parmi les problèmes auxquels l'UNTAET doit faire face, l'un des plus ardus est la mobilisation, au service de la reconstruction, de milliers de jeunes chômeurs esttimorais sans aucune qualification. L'existence d'une population importante de jeunes chômeurs offrirait en effet les conditions de troubles sociaux graves ${ }^{29}$, d'autant que nombre de ces jeunes furent fortement mobilisés par les mouvements de résistance à l'occupation indonésienne. La reconversion des jeunes auxiliaires des mouvements de libération nationale est, bien entendu, un problème auquel furent confrontées - de l'Algérie au Zimbabwe - toutes les sociétés post-coloniales issues d'une longue lutte armée. Le défi pour l'UNTAET est d'offrir de vrais emplois civils aux jeunes militants tout en démobilisant les combattants du Falintil et en les réinsérant dans la vie civile. Aujourd'hui, il est clair pour tous que la reconstruction est un processus extrêmement complexe qui répond à la concurrence d'agendas locaux, nationaux et internationaux divergents. Certaines sources potentielles de conflits sont d'ailleurs aisément repérables. Ainsi, les fonds qui affluent aujourd'hui au Timor-Oriental répondent aux besoins urgents de la reconstruction, mais amènent également le risque d'inégalités importantes, notamment entre habitants des villes et des campagnes. D'ailleurs, les troubles déclenchés par le programme d'embauches d'Est-Timorais par l'UNTAET résultaient précisément du sentiment que les gens de Dili y étaient injustement favorisés. À ces complexités, il faut ajouter la pléthore d'organismes donateurs et d'ONG internationales qui prennent désormais part au processus de reconstruction avec, tous, leurs propres intérêts, leurs propres caractéristiques structurelles et leur propre conception du développement. Ces organisations fortement structurées offrent un contraste saisissant avec les ONG est-timoraises, qui sont peu nombreuses et qui commencent seulement à se remettre de l'automne traumatisant de 1999. Autre facteur significatif de conflits possibles à l'avenir, le sentiment, répandu au sein des ONG esttimoraises, que les organisations internationales privilégient les expatriés de retour au pays, qui ont souvent plus d'atouts que ceux qui, restés au pays, ont vécu les exactions paramilitaires. On trouve, en effet, d'un côté les « locaux » qui ont vécu toute la période d'occupation indonésienne et, de l'autre, plusieurs générations d'Est-Timorais qui se sont établis, notamment, en Australie, au Portugal ou dans d'anciennes colonies portugaises tel Macao. Nombre d'émigrés sont aujourd'hui rentrés au Timor, parmi lesquels les plus visibles et les plus influents sont sans doute José Ramos Horta et João Carrascalao. Les émigrés parlent généralement l'anglais ou le portugais; ils appartiennent plutôt à l'ancienne génération et sont proches politiquement du Fretilin ou de l'Union démocratique du Timor-Oriental. Parmi les militants locaux, surtout les plus jeunes, en revanche, bon nombre ont été formés dans les universités indonésiennes. Ces derniers craignent de plus en plus que la reconstruction ne soit confisquée par les anciens de la diaspora anglophone ou lusophone. Si ce devait être le cas, l'unité de la direction nationale actuelle serait menacée par les tensions générationnelles, politiques et linguistiques. Comme d'autres, ce problème souligne l'exigence pour l'UNTAET de promouvoir une reconstruction participative qui n'avive pas les conflits qui divisent déjà la société est-timoraise. À cet égard, on peut porter au crédit de l'UNTAET la création d'un "Conseil de la réconciliation nationale ». Actif depuis début 2000, ce Conseil, qui compte onze membres représentatifs de la 
population est-timoraise dans sa diversité, est devenu l'un des principaux organismes consultatifs de l'administration transitoire. Sur le plan politique, des inquiétudes se sont fait jour au sein du CNRT car, au niveau des populations locales, une réelle volonté de mettre en place une administration parallèle à celle de l'UNTAET a été observée. Or, certains dirigeants du CNRT craignent qu'une telle structure soit dominée par le Fretilin aux dépens des autres partis politiques ${ }^{30}$. De telles inquiétudes rappellent les rivalités politiques très intenses ainsi que le climat de suspicion qui caractérisaient la scène politique est-timoraise avant la période de l'occupation indonésienne. Ces tensions, qui se font sentir aux niveaux local et national, sont, bien entendu, en rapport étroit avec différents facteurs internationaux. C'est ainsi que le Portugal prétend à un rôle majeur dans la reconstruction, comme ancienne métropole coloniale et comme important donateur actuel. En effet, la question du Timor-Oriental est, depuis de nombreuses années, l'une des principales préoccupations de la politique étrangère du Portugal, auquel elle a permis de jouer un rôle sur la scène internationale. Désormais, la stratégie d'influence portugaise passe par la promotion de l'escudo comme monnaie principale et du portugais comme langue officielle pour le Timor-Oriental. Des priorités économiques divergentes D'autres tensions, comparables à celles que nous venons d'évoquer, concernent la politique économique ; il semble qu'elles ne soient pas prêtes d'être dissipées. Les recommandations, analysées ci-dessus, de la mission commune d'évaluation de l'ONU et de la Banque mondiale vont dans le sens d'un Timor-Oriental économiquement libéral, en conformité avec les priorités néolibérales de la Banque mondiale. Les dirigeants est-timorais s'efforcent, certes, de convaincre la communauté internationale qu'ils adhèrent aux principes de l'économie de marché, mais les idées socialistes n'en restent pas moins puissantes, notamment au sein du Fretilin ${ }^{31}$. Pour voir les tensions possibles, il suffit d'envisager les conséquences pratiques du libéralisme au Timor-Oriental. Comme elles ne rencontraient aucun obstacle, les entreprises, notamment australiennes, ont vite tiré profit de l'« économie ouverte » pour fournir les travailleurs internationaux au Timor-Oriental en biens et services de toutes sortes. Certains Australiens sont même déjà parvenus à nouer des relations d'affaires avec des Est-Timorais proches de certains dirigeants du CNRT ${ }^{32}$. D'une manière générale, l'Australie a exercé une très grande influence sur les événements récents au Timor-Oriental par le travail de ses ONG, la coopération militaire et les relations d'affaires ; il en sera certainement de même, sur le plan politique comme sur le plan financier, à l'avenir aussi ${ }^{33}$. D'où des problèmes aisément prévisibles. Il est possible que la population est-timoraise conçoive de l'amertume si elle a le sentiment que la nouvelle économie du pays est contrôlée par l'étranger. Il est probable, sur la base des premières indications, que la politique économique néolibérale qui commence à être mise en œuvre donnera naissance à une reconstruction à deux vitesses qui accentuera les inégalités sociales. Disposant de ressources plus importantes, les émigrés réussiront probablement mieux que d'autres à nouer des relations d'affaires avec des investisseurs étrangers, et en tireront donc des bénéfices plus grands. En fait, une grande partie des Est-Timorais ne possède ni les moyens matériels ni les compétences nécessaires pour participer activement et durablement à la reconstruction économique. Bien souvent, en effet, leurs maisons et leurs biens ont été détruits au cours des exactions paramilitaires. Ces inégalités de richesses et de chances ont de profondes répercussions sur les sources potentielles de conflits que le TimorOriental connaîtra à l'avenir. En effet, ces inégalités sont combinées avec d'autres facteurs locaux, nationaux ou internationaux qui avivent encore les tensions au sein de 
la société est-timoraise. Il est possible aussi que des conflits éclatent autour de la question de la taille de la nouvelle administration est-timoraise. En effet, alors que la Mission commune d'évaluation souligne dans son rapport la nécessité de mettre en place une administration qui soit la plus ramassée possible, les dirigeants est-timorais, eux, sont soumis à la pression de la population qui aspire à voir naître au contraire une administration très développée susceptible de créer de nombreux emplois. Dans la deuxième partie de cet article, nous avons identifié quelques-uns des principaux problèmes auxquels la reconstruction en cours du Timor-Oriental doit faire face. Les tensions les plus fortes résultent, comme nous l'avons vu, de la combinaison de divisions profondes propres à la société est-timoraise et de l'héritage laissé par l'occupation coloniale. Pour y répondre, nous avons essayé de montrer qu'une reconstruction pacifiée exige une participation interactive, dans la durée, des populations locales au processus de restructuration. En effet, si la population esttimoraise n'a pas le sentiment de s'approprier la reconstruction de sa propre société, que ce soit sur le plan social, sur le plan politique ou sur le plan économique, alors les tensions vont rester très fortes et l'arrivée massive de capitaux étrangers ne fera que les accroître. Le scénario le plus inquiétant résulterait d'un processus de reconstruction imposé de l'étranger qui n'associerait pas les populations locales et qui ne réaliserait pas davantage une réconciliation nationale. Les défis qui attendent le Timor-Oriental: la construction d'une paix dans un Etat neuf aux conflits anciens Jusqu'à présent, nous nous sommes contentés d'étudier les problèmes auxquels le Timor-Oriental va devoir faire face à court terme. Aussi, dans la troisième et dernière partie de cet article, essayerons-nous plutôt d'identifier les facteurs qui peuvent permettre la réussite à long terme du processus de pacification. Comme dans tous les cas de conflits inscrits dans la longue durée, les tâches du long terme sont colossales, notamment parce qu'elles nécessitent « que l'on conçoive une paix qui ne présuppose pas que la guerre fait partie intégrante de la vie d'une société », pour reprendre les termes de Vivienne Jabri ${ }^{34}$. En fait, les principaux éléments qui permettent de concevoir une telle paix doivent s'intégrer dans un processus d'ensemble, qui relève de plusieurs niveaux de l'activité politique. C'est pourquoi dans la suite de cette troisième partie nous n'analyserons pas séparément ce qui se passe aux niveaux local, national et international. Au contraire, en effet, les dynamiques politiques les plus importantes sont à angle droit par rapport à ces niveaux. Nous mettrons donc l'accent sur ce que nous pourrions appeler des luttes "transversales ", c'est-à-dire mettant en jeu des forces politiques qui débordent les sphères établies de souveraineté et, de ce fait, remettent en question le cloisonnement qui maintient en place - souvent par la violence - l'ordre politique établi ${ }^{35}$. La nécessité de dépasser la violence : à propos du dialogue et de la différence Comme nous l'avons $\mathrm{vu}$, les années d'occupation indonésienne ont laissé la société est-timoraise en état de choc, travaillée par des tensions sociales fondamentales et par une défiance généralisée. Les différents groupes de la société est-timoraise vont donc certainement connaître de nouveaux conflits qui trouveront leur origine dans la revendication de biens de toutes sortes, notamment des terres, dans des désirs de vengeance ou encore dans des querelles ancestrales entre familles ou entre clans. De plus, le retour d'Indonésie des nombreux Est-Timorais qui y ont trouvé refuge avivera ces tensions en faisant émerger des prétentions concurrentes à la propriété des biens immobiliers et de la terre. Bien sûr, le retour au Timor-Oriental de ceux qui ont été membres des groupes paramilitaires anti-indépendantistes est un sujet plus explosif encore. Selon certaines sources en effet, il semble que les quelques membres de ces groupes qui sont revenus 
dans le territoire aient déjà été la cible de pressions violentes ${ }^{36}$. Il est vrai qu'aujourd'hui un terme a été mis à la plupart des exactions des milices antiindépendantistes, au moins sur le territoire du Timor-Oriental, mais le risque d'une recrudescence de la violence reste élevé. Faute d'un système judiciaire effectif, les indications de délinquance et de désordre généralisé ont désormais une fréquence alarmante $^{37}$. Dans certains districts de l'est du Timor-Oriental, de violents affrontements ont opposé des bandes de jeunes territorialement définies ${ }^{38}$. Enfin, il se peut également que les actes de violence augmentent avec le nombre de jeunes esttimorais au chômage qui se sentiront exclus du processus de reconstruction ${ }^{39}$. Si l'on adopte maintenant la perspective d'un éventuel processus de réconciliation, deux exigences éthiques ont une importance particulière. La première, banale, consiste à encourager le dialogue entre les acteurs du conflit puis à leur permettre de trouver des terrains d'entente en dépit de leurs différences. En effet, pour qu'un ordre social soit non seulement solide, mais aussi juste et équitable, il est indispensable que le dialogue l'emporte sur la violence. Pour reprendre les termes de Paul Ricœur, il s'agit « d'une part de porter les conflits au niveau du discours et, d'autre part, de ne pas les laisser dégénérer en actes de violence $»^{40}$. Plus concrètement, Wieviorka met en lumière le lien entre irruption de la violence et rupture (ou absence) du dialogue. Selon lui, en effet, la violence fait irruption lorsque les relations entre différents groupes d'une société se distendent très sérieusement ou bien, a fortiori, lorsqu'elles sont inexistantes ${ }^{41}$. Comme nous l'avons vu dans la première partie de cet article, les milices anti-indépendantistes entretenaient très peu de relations - et encore moins de dialogue - avec la petite partie de l'élite est-timoraise pro-indonésienne qui vivait à Dili ou à Djakarta : la violence en fut directement le résultat. En fait, l'expérience montre que la pacification exige la promotion de relations organiques et de rapports de dialogue entre individus et groupes qui, précédemment, avaient refusé l'interaction ou n'en avaient pas la possibilité. Il en résulte que l'un des défis majeurs de la pacification est l'attitude à adopter à l'égard des activistes les plus extrémistes dans les zones de conflit. Faut-il les exclure du processus de reconstruction pour rompre les relations qu'ils entretiennent avec des groupes plus modérés? Ou faut-il, au contraire, les associer à un projet de pacification aussi large que possible? Wieviorka est clairement partisan de la seconde solution. Selon lui, en effet, la recherche du dialogue est comparable «à la tâche d'une sociologie de la violence (...) [qui] est de montrer les médiations absentes, les systèmes de relations dont le manque ou l'affaiblissement créent l'espace de la violence $»^{42}$. Cependant, quelle que soit la solution retenue, la tâche à accomplir demeure colossale, car elle requiert des efforts minutieux étalés sur un temps qui dépasse bien souvent celui des initiatives planifiées. Cependant, le dialogue ne peut, seul, résoudre tous les problèmes que pose la reconstruction du Timor-Oriental. D'où le second aspect essentiel de la réconciliation : même si le dialogue entre les acteurs du conflit se révèle fructueux, il reste toujours des positions politiques qui ne sont pas solubles dans un compromis, voire ne peuvent pas être comprises par des personnes qui ne les vivent pas. Pour traiter ce « reste » irréductible et problématique, l'approche dialogique de la pacification doit se doubler d'une éthique de la différence. Celle-ci implique de résister au réflexe de réduire ce reste - qu'il représente une personne, un groupe, un point de vue ou une identité - aux termes d'un jugement extérieur ${ }^{43}$. Dans le cas du TimorOriental, l'éthique de la différence supposerait de créer les conditions nécessaires pour que les différents "ennemis " puissent dialoguer et vivre ensemble sans que leurs interactions dégénèrent en violence. Il ne s'agit pas ici de créer l'harmonie et l'amitié - 
certaines différences, certains antagonismes, peuvent en effet se révéler irréductibles mais plutôt de trouver un moyen pour que ces ennemis puissent cohabiter paisiblement. Dans le courant de cette dernière partie, nous allons étudier comment mettre en œuvre cette double éthique du dialogue et la différence dans le cadre de la pacification du Timor-Oriental. Il nous semble que trois grandes questions joueront un rôle déterminant dans le processus de passage à la paix : la réconciliation nationale ; la mise en place d'institutions pluralistes solides; et la promotion du bon voisinage international, notamment en ce qui concerne les relations entre le Timor-Oriental et l'Indonésie. Entre souvenir et oubli : justice et réconciliation nationale Pour donner une portée pratique aux concepts plutôt abstraits de dialogue et de différence, il faut analyser l'un des défis essentiels pour la société est-timoraise: la tâche, aussi indispensable que complexe et difficile, de la réconciliation nationale. Pour le moment, les dirigeants est-timorais sont essentiellement préoccupés par des questions de justice - de manière prioritaire, et fort compréhensible, il s'agit de poursuivre les coupables de violations des droits de l'homme - plutôt que par la réconciliation au sens large. La mission d'enquête sur le Timor-Oriental de la Commission indonésienne des droits de l'homme n'exclut pas que certaines sanctions puissent être prises contre ceux qui ont organisé les exactions de l'automne 1999. Cependant, bien que l'Indonésie cherche à réduire l'influence de l'armée au sein de son gouvernement, il est probable que la grande majorité des officiers de haut rang ne seront pas inquiétés. De fait, la fragilité même du processus de démocratisation en Indonésie limite sévèrement la capacité de poursuivre les auteurs de violations des droits de l'homme. Les réformateurs indonésiens se heurtent aux intérêts de l'armée et d'autres composantes de l'ancien régime, et doivent donc avancer à petits pas afin d'éviter une retour de bâton brutal. Ainsi, il est nécessaire de mettre en place un processus qui permette à la société esttimoraise d'accepter le souvenir des crimes dont elle a été la victime, sans qu'il soit tributaire de la justice indonésienne. Dès lors, un aspect essentiel de la réconciliation sera de la penser au-delà du seul châtiment des criminels. Selon Lederach, la réconciliation " correspond à un espace, un carrefour où peuvent se rejoindre le souci du passé et le souci de l'avenir $»^{44}$. Il s'agit donc d'apprécier comment le Timor-Oriental, considéré comme entité sociétale, pourrait accepter le souvenir de ses traumatismes et constituer les conditions d'un avenir plus paisible. C'est dans le domaine de la réconciliation que l'interaction entre promotion du dialogue et acceptation de la différence va jouer le rôle le plus crucial. Comment, en effet, envisager de construire un avenir de paix sur la base d'un passé de violence ? Plus précisément, comment traiter les souvenirs de douleur et de mort qu'ont engendrés des décennies d'occupation armée ? De quoi, et de quelle manière, faut-il se souvenir? Que faut-il oublier ? Est-il suffisant de punir les auteurs d'un crime pour que l'on puisse parler de justice? L'amnistie est-elle une solution? Si oui, pourquoi ? Si non, pourquoi pas? Par exemple, quel sort faut-il réserver aux Est-Timorais qui ont collaboré avec l'armée indonésienne? Enfin, comment le Timor-Oriental peut-il établir des relations saines avec l'Indonésie, sachant que cet Etat a colonisé son territoire vingt-quatre années durant et sachant également que la qualité de leurs relations jouera un rôle déterminant dans la mise en place d'une paix qui dure ? En fait, pour que le processus de réconciliation soit un succès, il devra combiner recherche de la justice et capacité de pardon. Bien sûr, un devoir de mémoire s'applique aux horreurs du passé, et de ce devoir résultent des actes nécessaires, dont les poursuites à l'encontre de personnes coupables de crimes contre l'humanité. L'instruction de tels dossiers a également la 
fonction essentielle d'offrir aux victimes une certaine reconnaissance publique de leur souffrance. Au-delà même des procédures judiciaires, le dévoilement et l'analyse historique des crimes du passé peuvent faciliter la guérison et la réhabilitation des victimes. Le devoir d'oubli, toutefois, n'est pas moins important. Selon Ricœur, «le devoir de mémoire est un devoir d'enseignement, alors que le devoir d'oubli est un devoir de dépassement de la colère et de la haine $»^{45}$. L'oubli, d'ailleurs, est un processus tout à fait naturel. Nous oublions, tous, que nous le voulions ou non. Il nous est impossible de nous souvenir de tout, ou même de mettre sur le même plan tout ce que nous avons vécu. La perception du présent et le souvenir du passé résultent ainsi tous deux d'une hiérarchisation, au terme de laquelle certains événements ou interprétations sont retenus en priorité, alors que d'autres prennent une importance secondaire ou sont tout à fait oubliés. Ce processus, inéluctable, est incontournablement politique. C'est en ce sens que la réconciliation doit, d'une manière ou d'une autre, passer à la fois par la mémoire et par l'oubli des doléances passées, fussent-elles l'effet des crimes les plus détestables. Cette part d'oubli ne revient ni à absoudre les criminels ni à nier leurs crimes, mais vise plutôt à rendre le passé suffisamment intelligible et compréhensible pour que des ennemis puissent, malgré tout, commencer à vivre en paix les uns à côté des autres ${ }^{46}$. Il ne suffit évidemment nullement d'affirmer le double devoir de mémoire et d'oubli, ni même de l'assumer dans l'action, pour rendre possible la réconciliation. Encore faut-il déterminer de quoi précisément il faut garder le souvenir, et ce qui, inversement, doit s'oublier. Et qui, d'ailleurs, doit en décider ? Ces questions sont sans réponse en dehors d'un processus dialogique inscrit dans la durée et ouvert le plus largement possible aux différentes composantes de la société. Pour ce qui est de la société est-timoraise, sortant péniblement de son état de choc, la thérapie au plus près des victimes est inséparable d'un tel dialogue national. De fait, certains signes semblent indiquer que le Timor-Oriental va plutôt dans le bon sens. La première visite d'un président de l'Indonésie dans le territoire depuis sa libération en est un bon exemple. Le 1er mars 2000, en effet, Abdurrahman Wahid se rend au Timor-Oriental et, en signe de réconciliation, Gusmão fait à son endroit un geste d'une exceptionnelle générosité : il lui donne l'accolade, puis déclare «il faut oublier le passé ». En réponse à ce geste, Wahid présente alors ses excuses " pour tout ce qui s'est passé » ${ }^{47}$. Gusmão et la plupart des autres dirigeants est-timorais parlent effectivement le langage de la réconciliation, attitude remarquable au regard des terribles injustices dont la population du TimorOriental, et souvent eux-mêmes à titre personnel, ont été victimes. Ramos Horta disait en mai 1999 : «le passé est le passé ». En dépit des exactions ultérieures, cette volonté de construire un avenir au-delà de la haine et de la vengeance semble toujours aussi forte ${ }^{48}$. La mise en place d'institutions démocratiques La violence de l'occupation indonésienne a laissé des plaies dont la cicatrisation sera longue et pénible. Par où commencer? Comme nous l'avons vu, la construction d'une paix qui dure au TimorOriental nécessite dialogue et réconciliation nationaux, qui exigent à leur tour des structures institutionnelles minimales qui, aujourd'hui, n'existent pas. Ainsi, nous avons vu que la combinaison du vide laissé par le départ précipité de l'administration coloniale et de la mise à l'écart des élites locales pendant l'occupation indonésienne avait favorisé la diffusion au Timor-Oriental d'une culture de la violence, notamment parmi les jeunes. En fait, comme c'est souvent le cas, l'origine de la violence porte ce que Wieviorka appelle, à juste titre, «la marque de la panne d'Etat $»^{49}$. Au TimorOriental cependant, même dans les domaines où l'appareil de l'Etat est encore en place, 
la transition va être source de problèmes épineux. Ainsi, jusqu'à l'automne 1999, les cadres administratifs et les membres des professions libérales étaient pour la plupart des Indonésiens, notamment dans les domaines de l'éducation et de la santé ${ }^{50}$. Il faudra donc du temps avant que des Est-Timorais formés et compétents puissent s'y substituer. De même, il sera long et difficile de mettre en place un système policier et judiciaire, ainsi qu'une société civile au plein sens du terme, comprenant en particulier les médias libres indispensables au dialogue national et à une véritable réconciliation. Nous avons vu que le développement de la société civile fut bloqué sous l'occupation indonésienne. Même les quelques médias et ONG est-timorais qui n'étaient pas interdits étaient sans cesse l'objet de tracasseries et elles devinrent la cible privilégiée des milices anti-indépendantistes ${ }^{51}:$ des représentants de la société civile qui n'hésitaient pas à s'exprimer en public furent assassinés, tandis que d'autres furent contraints de se réfugier dans la province indonésienne du Timor-Occidental ou d'entrer dans la clandestinité. Mais par où faut-il commencer pour restaurer ces structures institutionnelles? En fait, faute de structures existantes, seuls des mécanismes informels peuvent permettre d'entamer la reconstruction du Timor-Oriental. Dans ce contexte, l'Église et les ONG sont les mieux placées pour participer aux efforts de médiation et de réconciliation et aux campagnes d'information que ces efforts impliquent. En l'absence d'un système judiciaire, la résolution de questions individuelles reposera sur toute une gamme de médiations et de conciliations qui ne sont pas formellement judiciaires. Les acteurs internationaux, qui peuvent avoir une expérience pertinente tirée d'autres cas de reconstruction, ont également leur place. Enfin, un rôle accru pour les chefs coutumiers est-timorais, marginalisés dans le Sistem Pemerintahan indonésien, pourrait contribuer à combler le vide institutionnel. Cependant, ces mécanismes informels ne sauraient remplacer des institutions politiques efficaces, qui fonctionneront d'autant mieux sur la base d'une culture démocratique au sens le plus large, à savoir d'un mode de gouvernance fondé sur la transparence et la responsabilité. Sans une telle culture et les institutions qui l'expriment, la reconstruction risque de sombrer dans la violence. Il est vrai que ces conditions sont exigeantes, et rarement réunies dans les Etats nouvellement indépendants; au Timor-Oriental cependant, l'évolution récente est encourageante. Différents dirigeants est-timorais ont ainsi déclaré que la future constitution devrait se fonder sur le respect des droits de l'homme et sur les valeurs démocratiques pluralistes ${ }^{52}$. Bien sûr, la démocratie que connaîtra le Timor-Oriental prendra une forme singulière. Lorsque nous parlons de démocratie, en effet, nous ne faisons pas référence à un ensemble d'institutions et de procédures qui aurait été établis une fois pour toutes à partir de l'expérience des Etats-nations occidentaux. À notre sens, la démocratie est plutôt une culture, qui déborde largement les institutions et les procédures, dont l'essence est d'ouvrir les processus de décision au pluralisme, à la transparence et à la participation citoyenne ${ }^{53}$. Réconciliation et coexistence pacifique C'est dans le rapport du Timor-Oriental à l'Indonésie que s'expriment de manière paradigmatique les thèmes du dialogue, de la différence et de la réconciliation que nous avons analysés ici. Le plus imposant défi auquel le nouvel Etat soit confronté est en effet la normalisation des relations avec son puissant voisin. Or, les atrocités du passé, qui sont nécessairement la matrice de la reconnaissance réciproque, ne font-elles pas obstacle à l'éthique conjointe du dialogue et de la responsabilité ? La réconciliation et la réinsertion ne s'opposent-elles pas à la poursuite de la justice ? Peut-on simultanément amnistier des criminels et rendre à leurs victimes la maîtrise de leur destin? Quelle 
peut être, à l'appui de ces processus, la reconnaissance des différences au sein de la société indonésienne, notamment entre l'armée et le nouvel gouvernement civil ? Toutes ces questions pèsent lourdement sur l'avenir du Timor-Oriental, qui, comme micro-Etat émergeant, sera fortement dépendant de l'extérieur. Et, par la force de la géographie, qui juxtapose le Timor au vaste et instable archipel indonésien, Djakarta pèsera lourd dans cet " extérieur ». Les relations, aussi indispensables que périlleuses, entre l'Indonésie et le Timor-Oriental sont particulièrement exposées à deux facteurs de tension : le rapatriement des réfugiés est-timorais et l'administration de la frontière entre le Timor-Oriental et le Timor-Occidental. Pour ce qui est du rapatriement, force est de constater qu'un an après l'intervention de la force armée mandatée par l'ONU, plus de 100.000 Est-Timorais vivent encore en Indonésie, souvent dans des camps au Timor-Occidental. Il est vrai que leur retour est freiné par la propagande alarmiste des milices anti-indépendantistes, qui incite ceux qui pourraient revenir à croire qu'ils s'exposeraient alors à la violence et à la disette. En outre, la réinsertion des anciens fonctionnaires civils et militaires indonésiens, et a fortiori des 3.000 à 5.000 miliciens, est une question difficile et encore ouverte. L'administration de la frontière, quant à elle, sera problématique à plusieurs niveaux. On craint en Indonésie, notamment dans les milieux militaires, que le cas du Timor-Oriental ne crée un dangereux précédent, qui encourageraient d'autres provinces au statut contesté à revendiquer plus d'autonomie, voire l'indépendance. Les provinces les plus exposées sont peut-être Atjeh (au nord de Sumatra), Irian Jaya (la Papouasie occidentale) et, dans une moindre mesure, Riau (au sud de Singapour). Il faut souligner ici que, par le passé, l'Indonésie n'a pas hésité à orchestrer la déstabilisation du Timor-Oriental pour montrer aux indépendantistes le coût humain et matériel de la sécession. La stratégie pourrait resservir. Contre ce scénario catastrophique, seul le dialogue, facteur de confiance, peut faire barrage. À terme, l'ouverture de la frontière pourra y contribuer. Les deux parties ont ainsi tout à gagner aux relations commerciales : les Est-Timorais ont un besoin urgent de biens de consommation à bas prix, qui offrirait aux entreprises indonésiennes de nouveaux débouchés pour leurs produits. Mais, dans ce domaine comme dans les autres, les obstacles ne manquent pas. Les troupes indonésiennes et les milices anti-indépendantistes qui sont présentes dans la région ont intérêt à maintenir leur contrôle de la frontière, notamment compte tenu des bénéfices potentiels de la contrebande. Par ailleurs, certains Est-Timorais voient d'un très mauvais œil la possibilité d'une réconciliation avec l'Indonésie : en ouvrant la porte aux entreprises indonésiennes, elle exposerait à la concurrence leurs propres affaires, gérées en collaboration avec des entreprises australiennes, qui jouissent d'une situation de quasimonopole dans le territoire.

2 Dans cet article, nous avons passé en revue quelques-uns des défis politiques, économiques et sociaux - immédiats et à long terme - que le Timor-Oriental devra relever au cours de la transition vers l'indépendance. À cet égard, nous nous sommes particulièrement attachés à mettre en lumière les problèmes éthiques de la pacification dans ce territoire marqué, depuis des siècles, par les conflits et par la violence. Nous avons ainsi identifié quelques défis cruciaux, sans prétendre y avoir définitivement répondu. D'ailleurs, nous sommes convaincus que les problèmes politiques et éthiques que connaît le Timor-Oriental sont d'une ampleur telle qu'on ne peut partir de principes universels et a priori. Le discours et la pratique de l'éthique en situation de conflit, en effet, sont affaire de finesse et exigent une certaine modestie analytique. C'est sur le terrain, dans les luttes concrètes, que le Timor-Oriental doit se 
reconstruire : il en résulte que le commentaire doit également se situer à ce niveau. Parmi les défis auxquels le Timor-Oriental doit faire face dès aujourd'hui, l'un des plus cruciaux résulte de la conflictualité inscrite dans l'intersection de divisions sociales profondes et de l'héritage de la brutalité coloniale. La tâche de relever ce défi concerne les groupes rivaux de la société est-timoraise, mais aussi une série d'acteurs internationaux: gouvernements, entreprises multinationales, organismes donateurs bilatéraux et multilatéraux. Chacun, bien entendu, a ses propres contraintes organisationnelles et ses propres objectifs. S'il néglige cette complexité, le processus de reconstruction pourrait donc aviver les tensions existantes et entraver la quête de la paix et de la réconciliation. Pour éviter cet écueil, nous avons soutenu qu'il fallait que les organisations internationales et les dirigeants est-timorais associent les populations locales au processus de reconstruction. En effet, à défaut de la légitimité produite par des processus décisionnels véritablement participatifs, la recrudescence des conflits pourrait submerger la reconstruction nationale. À plus long terme, nous avons identifié deux autres défis pour la pacification et la réconciliation. Le premier consiste évidemment à établir des relations de dialogue et de confiance entre les acteurs du conflit. De manière moins évidente, cependant - mais tout aussi importante et peutêtre plus difficile - il faut également faire accepter à ceux-ci les limites inhérentes au dialogue : on n'élimine jamais complètement les différences et les animosités. Une reconstruction pacifique et durable du Timor-Oriental passe par l'acceptation de la différence, quitte, pour chacun, à vivre en paix aux côtés de ses ennemis. Pour que naisse l'espoir, il faut que les représentants des populations locales, les dirigeants nationaux, les organisations internationales - bref, tous ceux qui prennent part à la reconstruction du territoire - relèvent ce double défi. Cet espoir, c'est celui d'un avenir qui transforme les restes d'un passé de violence en modes plus paisibles d'interaction humaine.

\section{NOTES}

1. Les auteurs remercient, pour leurs commentaires critiques et leurs suggestions utiles, Mark Otter, Ralph Summy et Bill Tow, ainsi que les deux lecteurs anonymes de Cultures \& Conflits.

2. Richard Kearney, « Narrative and the Ethics of Remembering », in R. Kearney \& M. Dooley (dir.), Questioning Ethics : Contemporary Debates in Philosophy, Londres, Routledge, 1999, p. 18.

3. E. Tee, «It's a Difficult Birth of a New Nation », The Straits Times [Singapour], 13 février 2000.

4. « 600 Listed as Dead, but Actual Toll Higher », The Straits Times, 4 mars 2000.

5. Amnesty International, «Statement of Human Rights Watch and Amnesty International on the Decolonization of East Timor », 6 octobre 1999, p. 1. (http:// www.amnestyusa.org/news/1999/ easttimor10061999.html). 
6. Voir Gilles Bataillon, « Analyser le chaos », in « Survivre. Réflexions sur l'action en situation de chaos », sous la direction de Gilles Bataillon, Cultures \& Conflits, $n^{\circ} 24-25$, printemps 1997.

7. John Paul Lederach, Building Peace : Sustainable Reconciliation in Divided Societies, United States Institute of Peace Press, Washington DC, 1997, p. xvi.

8. J. Dunne, Timor : A People Betrayed, Sydney, ABC Books, 1996.

9. J. Dunne, Timor : A People Betrayed, ibid.

10. P. Carey, «Introduction » et John G.Taylor, « The Emergence of a Nationalist Movement in East Timor », in P. Carey \& G. Carter Bentley (dir.), East Timor at the Crossroads : The Forging of a Nation, Honolulu, University of Hawaii Press, 1995.

11. Didier Bigo, « Guerres, conflits, transnational et territoire », in Bertrand Badie \& Marie-Claude Smouts (dir.), «L'international sans territoire », Cultures \& Conflits, n²1-22, été 1996, pp. 2- 6.

12. William E. Connoly, The Ethos of Pluralization, Minneapolis, University of Minnesota Press, 1995, p. xxii.

13. Sur ce tournant dans la stratégie des mouvements de résistance, voir Carey, « Introduction » et Taylor, « The Emergence of a Nationalist Movement », op. cit. 14. À ce sujet, voir Stephen R. Shalom, Noam Chomsky \& Michael Albert, « East Timor : Questions and Answers ", Z Magazine, février 2000. (http://www.zmag.org/ CrisesurEvts/ Timor/ qanda.htm - consulté en février 2000).

15. João Mariana de Sousa Saldanha, The Political Economy of East Timor Development, Djakarta, Pustaka Sinar Harapan, 1994.

16. Voir George Aditjondro, In the Shadow of Mount Ramelau : The Impact of the Occupation of East Timor, Leyde (Pays-Bas), Indonesian Documentation and Information Centre, 1994 ; ainsi que Carey, «Introduction », pp 12-14.

17. Aditjondro, In the Shadow of Mount Ramelau, op. cit.

18. Sur le Sistem Pemerintahan (que l'on peut traduire par «système d'administration moderne »), voir Sousa Saldanha, The Political Economy of East Timor Development, op. cit.

19. Rapport de la CIA cité par Shalom, Chomsky \& Albert, « East Timor : Questions and Answers ", op. cit.

20. Michel Wieviorka, « Le nouveau paradigme de la violence », in Michel Wieviorka (dir.), « Un nouveau paradigme de la violence? », Cultures \& Conflits, $n^{\circ} 29-30$, été 1998, p. 8.

21. Parmi les meilleures informations sur la crise au Timor, on peut consulter les rapports du Carter Center, ONG américaine qui s'efforce, sous l'égide de l'ancien Président Jimmy Carter, de promouvoir la démocratie. Les rapports sont consultables sur http://www.cartercenter.org/ reports.html.

22. M. Dodd, « Saying by Numbers : Mummy Where are You? », Sydney Morning Herald, 8 mars 2000 .

23. E. Tee, «It's a Difficult Birth », op. cit.

24. C. Barr, «For E Timor, Half a Billion to Build a Nation », The Christian Science Monitor, 21 décembre 1999.

25. «Rebuilding East Timor at the Local Level », communiqué de presse de la Banque mondiale, 22 février 2000 (cf. http://www.worldbank.org/).

26. J.-P. Lederach, Preparing for Peace, op. cit., p. 20.

27. À ce sujet, on peut se référer aux critiques émises par les ONG spécialisées dans les questions de développement à l'encontre des institutions financières internationales à 
l'occasion de la dernière assemblée annuelle de la Conférence des Nations unies sur le commerce et le développement : voir Ranjit Dev Raj, « UNCTAD Tells NGOs to Fight it Out with Governments », Inter Press Service, 15 février 2000.

28. E. Tee, «It's a Difficult Birth », op. cit.

29. À ce sujet on peut notamment se référer à une correspondance de Dili diffusée par les actualités télévisées de la BBC le 21 février 2000 ; ainsi qu’à J. Jolly, « Goodwill Ebbs as Unemployed Grow Restless », South China Morning Post, 18 février 2000 ; « East Timor Not Out of the Woods? Not Quite?», The Straits Times, 23 février 2000. 30. Entretien des auteurs avec le militant social Ibu Ade Rostina Sitompul, réalisée à Djakarta le 26 janvier 2000.

31. George Aditjondro, Self-Determination Under Globalization : Timor Loro Sa'e's Transformation from Jakarta's Colony to a Global Capitalist Outpost, inédit, Université de Newcastle, Royaume-Uni, décembre 1999, p. 5.

32. M. Dodd, « Open for Business, but a While before the Good Times Roll », Sydney Morning Herald, 7 mars 2000.

33. Brian Hallet \& Ralph Summy, «Introduction : Past, Present, and Future of the East Timor Struggle », Pacifica Review, 12 (1), février 2000, p. 14.

34. Vivienne Jabri, Discourses on Violence : Conflict Analysis Reconsidered, Manchester, Manchester University Press, 1996, p. 154.

35. Sur la «transversalité », voir : David Campbell, « Political Prosaics, Transversal Politics, and the Anarchical World ", in Michael J. Shapiro \& Hayward R. Alker (dir.) Challenging Boundaries : Global Flows, Territorial Identities, Minneapolis, University of Minnesota Press, 1996 ; ainsi que Roland Bleiker, Popular Dissent, Human Agency and Global Politics, Cambridge, Cambridge University Press, 2000.

36. E. Tee, «It's a Difficult Birth», op. cit.

37. Nous nous référons à « East Timor Not Out of the Woods? Not Quite? », op. cit. ; ainsi qu'à l'entretien déjà cité avec Ibu Ade Rostina Sitompul.

38. «East Timor Not Out of the Woods? Not Quite?» op. cit.

39. Reportage télévisé diffusé par la BBC, op. cit.; Jolly, « Goodwill Ebbs », op. cit.

40. Paul Ricœur, «Imagination, Testimony and Trust », in Kearney \& Dooley, Questioning Ethics, p. 12.

41. Michel Wieviorka, «Le nouveau paradigme de la violence », op. cit., p. 15.

42. Michel Wieviorka, «Le nouveau paradigme de la violence », op. cit., pp. 15-16.

43. Pour une analyse théorique et empirique plus approfondie de la possibilité de combiner éthiques du dialogue et de la différence, voir Roland Bleiker, «Identity and Security in Korea », inédit. Parmi les références fondamentales pour l'éthique de la différence, on peut se référer à : Emmanuel Levinas, Totalité et infini. Essai sur l'extériorité, La Haye, M. Nijhoff, 1961 ; Emmanuel Levinas, « Paix et proximité », in Jacques Rolland (dir.), « Emmanuel Levinas », Les Cahiers de la Nuit Surveillée, $n^{\circ} 3$, Lagrasse, Verdier, 1984 ; Iris Marion Young, Justice and the Politics of Difference, Princeton (NJ), Princeton University Press, 1999. Pour l'application de ce cadre éthique aux relations internationales, voir : David Campbell, National Deconstruction : Violence, Identity and Justice in Bosnia, Minneapolis, University of Minnesota Press, 1998 ; Vivienne Jabri, « Explorations of Difference in Normative International Relations ", in V. Jabri \& E. O'Gorman (dir.), Women, Culture and International Relations, Boulder (Col.), Lynne Rienner, 1999 ; Michael J. Shapiro, Violent Cartographies : Mapping Cultures of War, Minneapolis, University of Minnesota Press, 1997. 
44. J.-P. Lederach, Building Peace : Sustainable Reconciliation in Divided Societies, op. cit., p. 27.

45. Paul Ricœur, « Memory and Forgetting », in R. Kearney \& M. Dooley (dir.), Questioning Ethics, op. cit., p. 11.

46. Pour une analyse plus approfondie des tensions politiques entre mémoire et oubli, voir de Roland Bleiker, « Forget IR Theory », Alternatives, 22(1), 1997, pp. 57-86 ; et, s'agissant du cas particulier du génocide nazi, « Give it the Shade : Paul Celan and the Politics of Apolitical Poetry », Political Studies, 47, 1999, pp. 661-676.

47. Ian Timberlake \& John Martinkus, « Wahid says sorry in emotional visit to devastated Dili », The Canberra Times, 1er mars 2000, p. 1.

48. José Ramos Horta, « From Kissinger to Albright : The US and East Timor », Pacifica Review, 12 (1), février 2000, p. 77.

49. Michel Wieviorka, «Le nouveau paradigme de la violence », op. cit., p. 22.

50. Amnesty International, «Statement on the Decolonization of East Timor », p. 1.

51. Voir les rapports hebdomadaires 7 à 9 du Carter Center.

52. Voir l'entretien avec Gusmão : « I Don't Feel Prepared to Lead this Country », Time Asia, 20 mars 2000, p. 12.

53. Campbell (David), National Deconstruction : Violence, Identity and Justice in Bosnia, op. cit., pp. 165-208.

INDEX

Index géographique : Asie du Sud-Est

Mots-clés : gestion de conflits, maintien de la paix, sortie de conflits 